\title{
Multiperiodicity in the light variations of the $\beta$ Cephei star $\beta$ Crucis
}

\author{
J. Cuypers ${ }^{1}$, C. Aerts ${ }^{2}$, D. Buzasi ${ }^{3}$, J. Catanzarite ${ }^{4}$, T. Conrow ${ }^{5}$, and R. Laher ${ }^{6}$ \\ 1 Koninklijke Sterrenwacht van België, Ringlaan 3, 1180 Brussel, Belgium \\ 2 Instituut voor Sterrenkunde, Katholieke Universiteit Leuven, Celestijnenlaan 200B, 3030 Heverlee, Belgium \\ e-mail: Conny.Aerts@ster. kuleuven.ac.be \\ 3 Department of Physics, US Air Force Academy, Colorado Springs, CO 80840, USA \\ e-mail: Derek.Buzasi@usafa.af.mil \\ ${ }^{4}$ Interferometry Science Center, California Institute of Technology, Pasadena, CA 91125, USA \\ e-mail: jcatanz@ix.netcom.com \\ 5 Infrared Processing and Analysis Center, Mailstop 100-22, California Institute of Technology, \\ Pasadena, CA 91125, USA \\ e-mail: tim@ipac.caltech.edu \\ ${ }^{6}$ SIRTF Science Center, Mailstop 314-6, California Institute of Technology, Pasadena, CA 91125, USA \\ e-mail: laher@ipac.caltech.edu
}

Received 11 April 2002 / Accepted 20 June 2002

\begin{abstract}
High-resolution spectroscopic observations have led recently to the discovery that the $\beta$ Cephei star $\beta$ Crucis (Mimosa) is multiperiodic with at least three non-radial pulsation modes. Data obtained by the star tracker of the WIRE satellite have now allowed us to confirm this multiperiodicity in the light variations. A total of 5 million observations covering 17 days was analysed and the three main periods we find in this work are in perfect agreement with the results derived from the line profile variations. The photometric amplitudes are small (3, 2.7 and 0.6 millimag for the dominant modes), but this is not surprising in view of the mode identifications derived earlier from the line profiles. Additional periods of low-amplitude modes (between $0.2-0.3 \mathrm{mmag}$ ) are also derived, including one suggested earlier by the radial velocity data.
\end{abstract}

Key words. stars: early-type - stars: oscillations - stars: individual: $\beta$ Crucis

\section{Introduction}

Light and radial velocity variations in the bright southernhemisphere star $\beta$ Crucis (Mimosa, HD 111123) were first observed long ago (Wright 1909; Pagel 1956; Van Hoof 1962). Pagel (1956) classified the star as a $\beta$ Cephei star and noted some amplitude variations in the radial velocities. Heintz (1957) first noted indications that $\beta$ Crucis was binary.

Cuypers (1983) performed a period analysis of all photometric data available at that time. These observations were collected in the period 1959 to 1975 . A main pulsation period of $0.1911846 \mathrm{~d}$, corresponding to a frequency of $5.23055 \mathrm{c} \mathrm{d}^{-1}$ (hereafter called $f_{1}$ ) was finally retained. The daily aliases were extremely strong in all these data sets and led other authors to claim frequencies of 4.23 or $6.23 \mathrm{c} \mathrm{d}^{-1}$. Some additional variability seemed present in the data analysed by Cuypers (1983), but no unambiguous secondary period could be established.

Send offprint requests to: J. Cuypers, e-mail: Jan.Cuypers@oma . be
Only the frequency $2.7874 \mathrm{~cd}^{-1}$ was found marginally significant in both the radial velocity and the light variations.

High-resolution spectroscopic data spanning almost 13 years were analysed by Aerts et al. (1998) (hereafter called Paper I). The binary nature of $\beta$ Crucis was again confirmed in Paper I and an unambiguous orbital period of 5.0 years was for the first time deduced from the variations of the average radial velocities. For the other orbital elements we refer to Paper I. Multiperiodicity was clearly visible in the line profile variations. Two new frequencies $\left(f_{2}=5.958666 \mathrm{~cd}^{-1}\right.$ and $f_{3}=5.472165 \mathrm{c} \mathrm{d}^{-1}$ ) were reported in Paper I, in addition to the one already found present in the light variations. These two additional frequencies clearly showed up in the moment variations of the $\mathrm{Si}$ III $\lambda \lambda 4567 \AA$ triplet. Additional variability was suspected but could not be confirmed due to the poor duty cycle of the spectroscopic data.

Aerts et al. (1998) have used the moment method to identify the pulsation modes in $\beta$ Crucis. The basic principle 


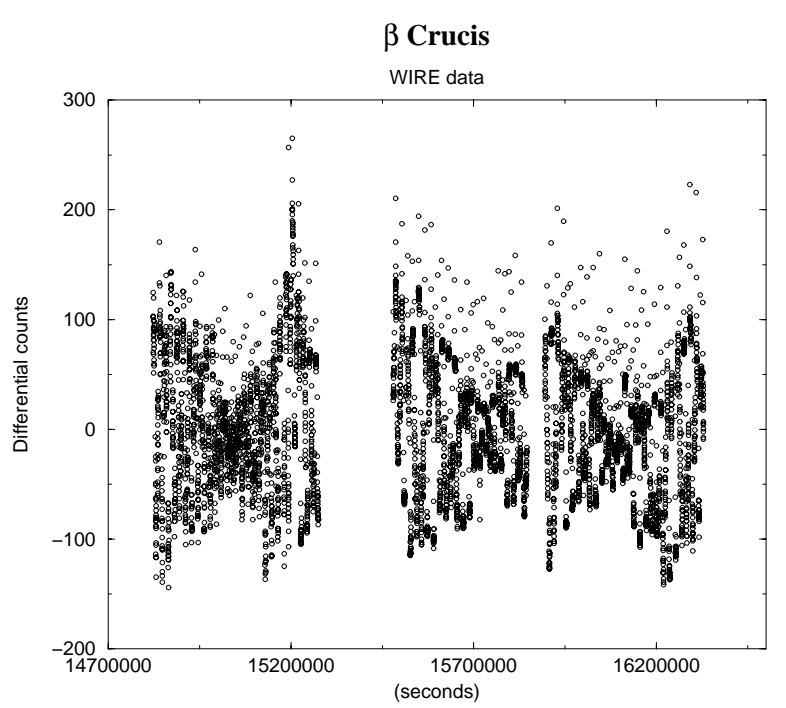

Fig. 1. All binned WIRE data of $\beta$ Crucis (each point represents about 1000 measurements).

of this quantitative identification method is to compare the amplitudes of the first three moment variations of the line profile of a selected spectral line with predicted values based upon pulsation theory. A discriminant function of the spherical wavenumbers $(\ell, m)$ is defined in such a way that the most likely mode minimizes the discriminant. At present, this method is the best one available for slow rotators. For a full description of the method we refer to Aerts et al. (1992) and Aerts (1996). The mode identification performed in Paper I by means of the moment method resulted in the conclusion that none of the modes is radial. The newly detected frequencies $f_{2}$ and $f_{3}$ seemed to correspond to modes with $\ell \geq 3$.

$\beta$ Crucis was observed intensively by the star tracker of the WIRE satellite. Buzasi et al. (2000), Buzasi (2002), Poretti et al. (2002) and Cuypers et al. (2002) described how this star tracker was used for asteroseismology and other variable star observations. Here we present the results derived from the $\beta$ Crucis data and we comment on the amplitudes in view of the mode identifications obtained earlier in Paper I.

\section{Frequencies in the WIRE data}

$\beta$ Crucis was observed by the WIRE satellite during 17 days, with one large time gap of about 2.4 days and a shorter second one of about 9 hours. At a rate of $10 \mathrm{~Hz}$ about 5 million measurements of the star were collected. These data came in blocks of about 38 min per 96 min orbit. Near the end of the first data interval there was a sudden brightening of $0.01 \mathrm{mag}$ of the star for the duration of about one orbital block (see Fig. 1). This is probably an instrumental effect, but the cause is still unknown. These data were disregarded in the work presented in this paper.

Many features which correlated with the orbital period of the satellite were seen in the data and correcting for these was not easy a priori. However, a first look at the data immediately reveals a clear beat pattern: at least two frequencies must be present (Fig. 1). Several binning schemes were used, but, since

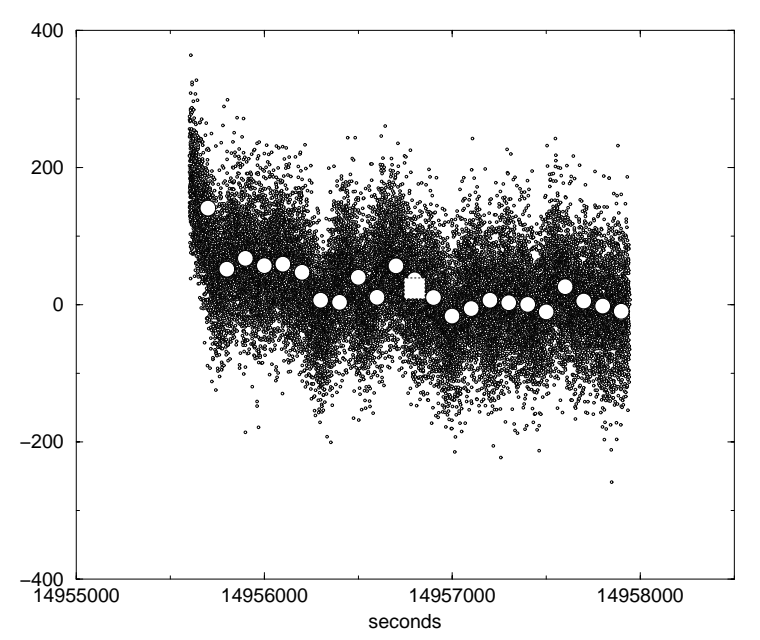

Fig. 2. One orbit of WIRE data of $\beta$ Crucis. Circles indicate binned data; the square is the average value for this orbit as used in the period analysis (differential counts).

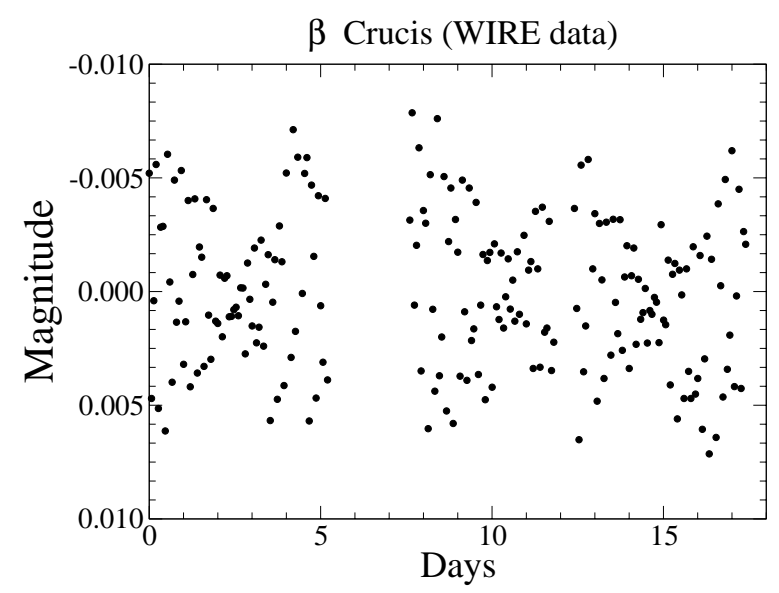

Fig. 3. The light curve of the WIRE data of $\beta$ Crucis used for the frequency analysis. Each data point is the mean value of the observations over one satellite orbit.

the expected periods of the light variations ( 4.6 hours) are considerably longer than the orbital period of the satellite, we decided to use only the mean value of the observations for each orbit (Fig. 2). This resulted in 214 very high quality data points for the frequency analysis (Fig. 3). The average value of the standard deviations in each group was about 0.0044 mag. This can be considered as the observational error on a single integration, while the photon noise is about $0.0021 \mathrm{mag}$. Since other sources of error are surely present, as already indicated by Poretti et al. (2002), the standard error of the mean of the 214 bins ( $0.00003 \mathrm{mag}$ ) cannot be considered as the observational error of these data. The rms of the residuals after the frequency fit will be a more realistic estimate.

A straightforward frequency analysis of the 214 points immediately led to two frequencies: $5.228 \mathrm{c} \mathrm{d}^{-1}$ and $5.477 \mathrm{c} \mathrm{d}^{-1}$ (panels (a) and (b) of Fig. 4). They are extremely close to the frequencies $f_{1}$ and $f_{3}$ found from the line profile variations in Paper I. A third frequency was also identified without ambiguity: $5.956 \mathrm{c} \mathrm{d}^{-1}$ (panels (c) and (d) of Fig. 4). This third frequency is, within the errors, equal to the frequency $f_{2}$ of the 
$\beta$ Crucis WIRE data

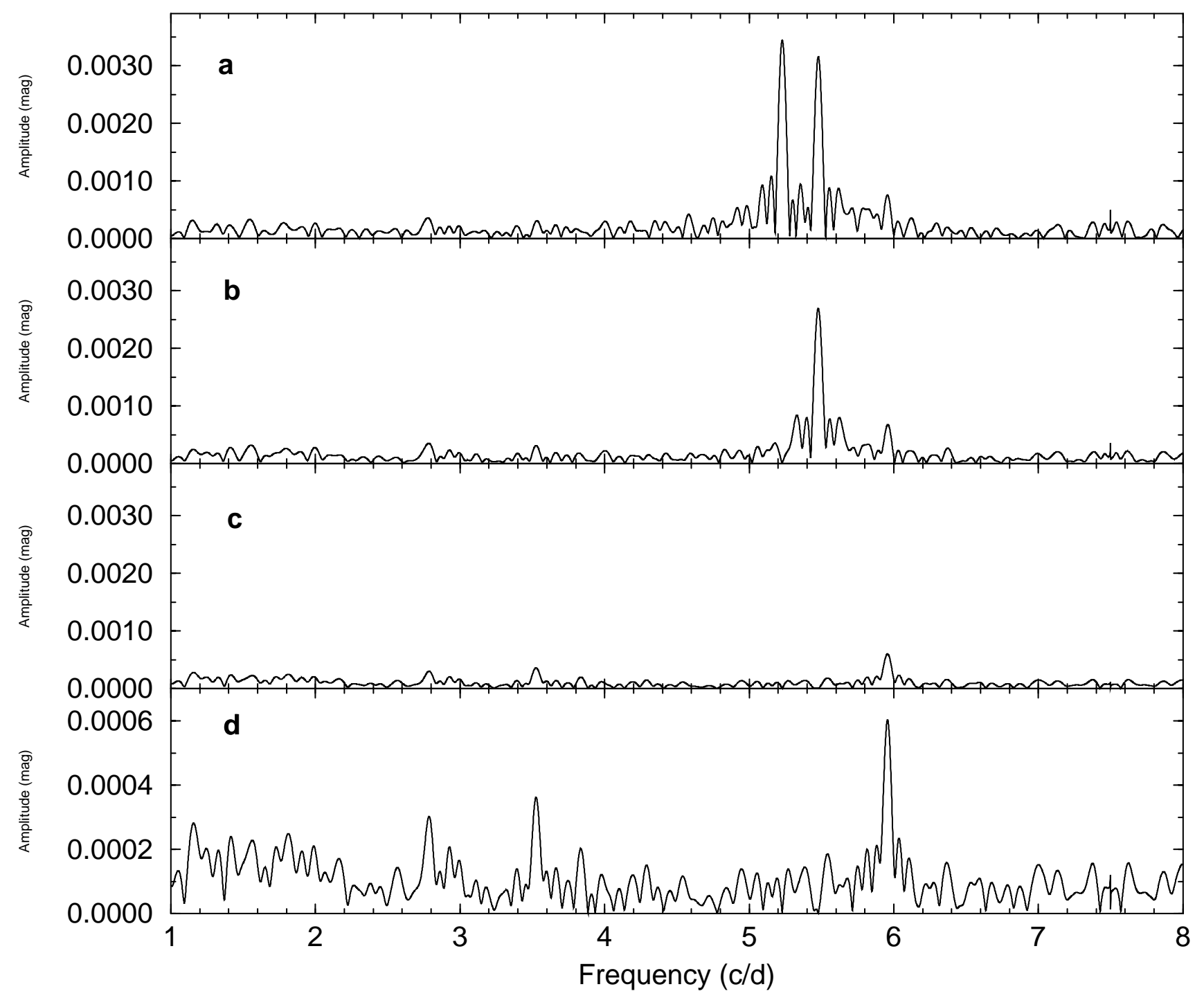

Fig. 4. Lomb-Scargle periodograms of the WIRE data of $\beta$ Crucis without prewhitening a), after prewhitening for $f_{1} \mathbf{b}$ ) and after prewhitening for $f_{2}(\mathbf{c})$ and $\left.\mathbf{d}\right)$ ). In panel d) the periodogram of panel c) is plotted again, but the scale of the $y$ axis is changed for clarity.

spectroscopic analysis in Paper I. We particularly remark that the amplitude of this variation is only $0.0006 \mathrm{mag}$.

After prewhitening there is still variability present in the residuals, which can also already be seen in panel (d) of Fig. 4. Although the amplitudes of the frequencies $f_{4}=3.527 \mathrm{~cd}^{-1}$ and $f_{5}=2.785 \mathrm{~cd}^{-1}$ are not high above the noise level, estimated by us to be smaller than 0.0002 mag in this frequency range, we have confidence in the reality of these two additional frequencies because $f_{5}$ coincides precisely with the secondary frequency suggested earlier by Cuypers (1983) using the radial velocity data he had at his disposal. The residual $\mathrm{rms}$ of the least-squares solution with the 5 frequencies is $0.0012 \mathrm{mag}$. This value is comparable to the residual rms of $0.0015 \mathrm{mag}$ found by Poretti et al. (2002) in WIRE observations of $\theta^{2}$ Tau.

The 3 main frequencies of $\beta$ Crucis are independently identified in the second, 9 day, data block. In the first data block, $f_{1}$ and $f_{3}$ could not be separated, since its length was only
5 days. We obtained the same frequencies using the non-binned data.

A summary of the frequencies found in the available data of $\beta$ Crucis is given in Table 1 .

\section{Amplitudes and mode identifications}

Although no multi-colour photometry over a long time interval is available, we can check whether the amplitudes found here and in the literature (summarized in Table 2) are in agreement with the previous spectroscopic mode identifications $(\ell=1$ for $f_{1}, \ell=3$ for $f_{2}, \ell=4$ for $f_{3}-$ see Paper I). We have used the relative amplitude estimates as a function of wavelength and mode as described by Heynderickx et al. (1994) with the parameters for $\beta$ Crucis as given in Paper I. In Fig. 5 we show the amplitude behaviour for the frequency $f_{3}$ as a function of wavelength for the stellar parameters of $\beta$ Crucis. 
Table 1. Overview of frequencies found in the $\beta$ Crucis data. The column Radial velocities gives the results of a frequency search in historical data as given by Cuypers (1983). The errors indicated were calculated as e.g. in Montgomery \& O’Donoghue (1999).

\begin{tabular}{|c|c|c|c|c|c|c|}
\hline \multirow{2}{*}{$\begin{array}{c}\text { Frequency } \\
\left(\mathrm{cd}^{-1}\right)\end{array}$} & \multicolumn{2}{|c|}{ Photometry } & \multicolumn{2}{|c|}{ Spectroscopy } & \multirow[t]{2}{*}{ Hipparcos } & \multirow[t]{2}{*}{ WIRE } \\
\hline & Shobbrook (1979) & Cuypers (1983) & Radial velocities & Paper I & & \\
\hline$f_{1}$ & 5.2301 & $5.23059(2)$ & 5.2326 & 5.23055 & $5.23046(3)$ & $5.228(1)$ \\
\hline$f_{2}$ & - & - & - & 5.95867 & - & $5.956(7)$ \\
\hline$f_{3}$ & - & - & - & 5.47217 & $5.47732(8)$ & $5.477(2)$ \\
\hline$f_{4}$ & - & - & - & - & - & $3.527(13)$ \\
\hline$f_{5}$ & - & $2.7872(1)$ & 2.7874 & - & - & $2.785(13)$ \\
\hline
\end{tabular}

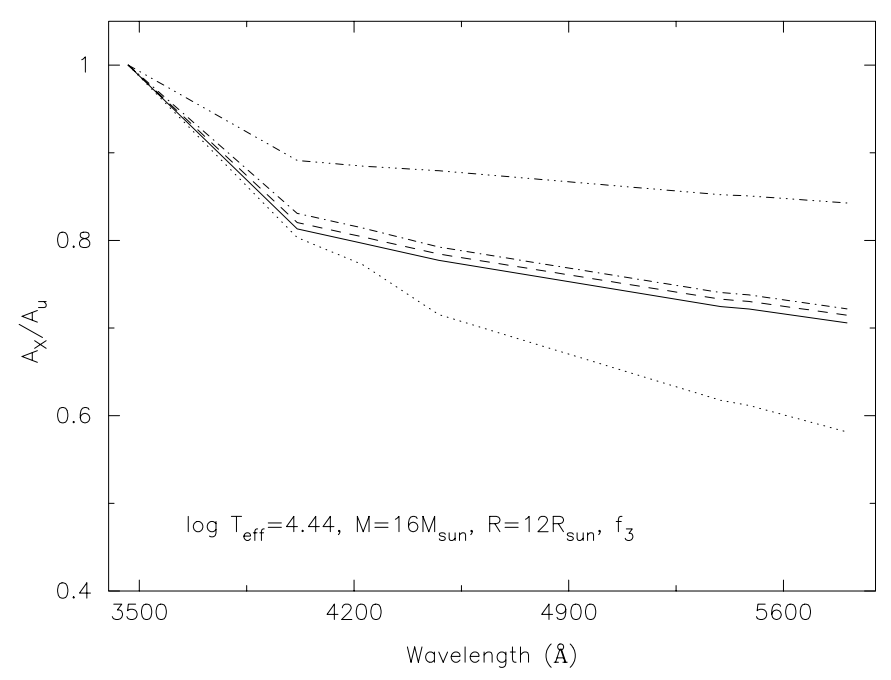

Fig. 5. Amplitude ratios as a function of wavelength for the 7 filters of the Geneva system with respect to $U$ filter for the stellar parameters of $\beta$ Crucis and for the frequency $f_{3}$. The meaning of the different line symbols is the following: full line $\ell=0$, dashed line $\ell=1$, dashed-dot line $\ell=2$, dotted line $\ell=3$, dashed-dot-dot-dot line $\ell=4$.

Different line symbols correspond to different degrees $\ell$. The amplitude behaviour shown in Fig. 5 is qualitatively the same for all three frequencies $f_{1}, f_{2}$ and $f_{3}$. The spectral response of the WIRE star tracker is approximately equivalent to a $V+R$ filter, while the usable photometric observations obtained by Van Hoof (1962), and re-analysed by Cuypers (1983) were done with a $U$ filter. For all modes a decrease of the amplitudes towards the red is expected, as can be seen from Fig. 5 .

The amplitude of $f_{1}$ as observed by WIRE ( $\left.0.0031 \mathrm{mag}\right)$ is smaller than the expected $70 \%$ of the $U$ amplitude of $0.012 \mathrm{mag}$ for a mode $\ell=1$, but other $\ell$-values do not give a significantly better result. The amplitude of 0.010 mag of $f_{1}$ in the Hipparcos data (ESA 1997) of $\beta$ Crucis is about $80 \%$ of the $U$ amplitude and in perfect agreement with the $\ell=1$ mode identification in Paper I.

It is unsurprising that $f_{2}$, with a WIRE amplitude of only $0.0006 \mathrm{mag}$, was never identified in photometric data from the ground, since an $\ell=3$ mode will have at most an amplitude of $0.002 \mathrm{mag}$ in the visible or in $U$.

On the other hand, the decrease in amplitude towards the red is not very large for the $\ell=4$ mode shown in Fig. 5. Since the WIRE amplitude of $f_{3}$ is $0.0027 \mathrm{mag}$, the corresponding
Table 2. The amplitudes of the light variations of $\beta$ Crucis derived from a multi-frequency least squares fit. The amplitudes of the $U$ data were recalculated, since the spline fit used by Cuypers (1983) overestimated the amplitude. The errors indicated were calculated as e.g. in Montgomery \& O’Donoghue (1999).

\begin{tabular}{cccc}
\hline \hline $\begin{array}{c}\text { Frequency } \\
\left(\mathrm{c} \mathrm{d}^{-1}\right)\end{array}$ & $U$ data & $\begin{array}{c}\text { Hipparcos } \\
\text { mag }\end{array}$ & WIRE \\
\hline$f_{1}$ & $0.012(1)$ & $0.010(1)$ & $0.0031(1)$ \\
$f_{2}$ & - & - & $0.0006(1)$ \\
$f_{3}$ & - & $0.004(1)$ & $0.0027(1)$ \\
$f_{4}$ & - & - & $0.0003(1)$ \\
$f_{5}$ & $0.003(1)$ & - & $0.0003(1)$ \\
\hline
\end{tabular}

predicted $U$ amplitude will be about 0.004 mag, which probably explains why $f_{3}$ was not previously identified in the rather noisy available $U$ data. However, a similar amplitude should be present in the visible region if the $\ell=4$ identification is correct. Examination of the Hipparcos data (ESA 1997) shows that after prewhitening for $f_{1}$, a clear secondary, but only marginally significant frequency is present at $5.4773 \mathrm{~cd}^{-1}$ (see Fig. 6). This is extremely close to the frequency $f_{3}$ and the amplitude $(0.004 \mathrm{mag})$ is indeed in good agreement with the $\ell=4$ mode identification proposed in Paper I from the moment method.

The nature of the frequencies $f_{4}$ and $f_{5}$ is not identified at the moment. We have included their amplitudes in the summary table of the amplitudes (Table 2) for reasons of completeness.

\section{Conclusions}

It is very fortunate that the asteroseismological programme of the star tracker of the WIRE satellite included the massive main sequence pulsator $\beta$ Crucis. The multiperiodicity of this star, previously known only from an analysis of high-resolution spectroscopic data, could be confirmed in the light variations of this object. Moreover, the amplitudes of the light variations as observed by WIRE are in agreement with the mode identifications derived from the spectroscopic data. We have derived three unambiguous common frequencies in spectra and in photometry now, and two additional very likely ones. Very few $\beta$ Cephei stars exist with five or more non-radial mode detections. Moreover, we have obtained compatibility between the spectroscopic mode identification and the amplitudes derived from the space photometry. $\beta$ Crucis is therefore at present one 


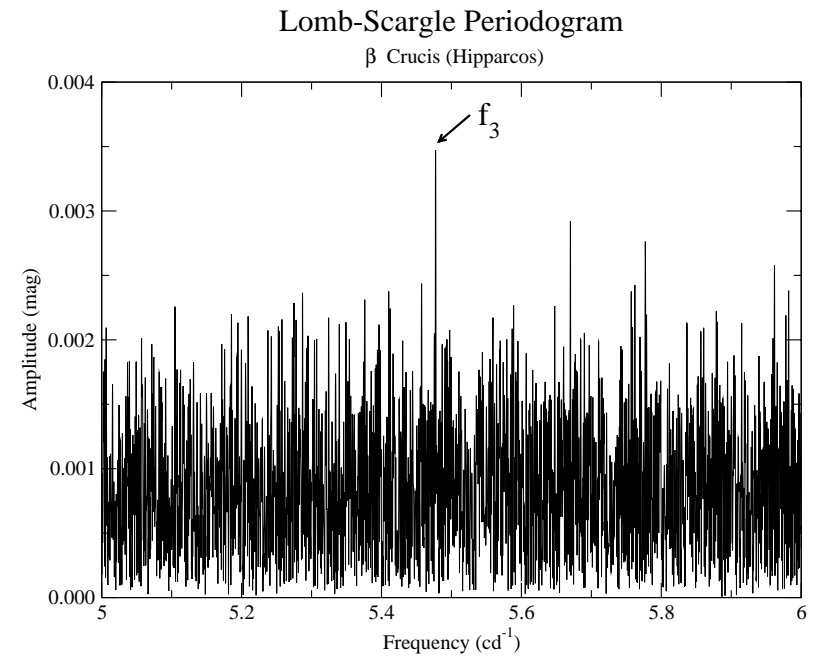

Fig. 6. Lomb-Scargle periodogram for the Hipparcos observations of $\beta$ Crucis prewhitened by $f_{1}$. Frequency $f_{3}$ at $5.4773 \mathrm{~cd}^{-1}$ can be identified easily.

of the best candidate $\beta$ Cephei stars on which to perform seismology. It is also one of the most massive $\beta$ Cephei stars known to date. The frequencies we provide, together with the mode identifications done in Paper I, should be explored further with the aim of detailed physical modeling of the internal structural parameters of the star.

Our findings lead to the following more general comments. When spectra with a $S / N$ ratio above 200 are available and they cover all phases of the oscillation cycle well (at least about 20 well distributed points per cycle), the line profile studies will reveal the non-radial modes excited in the $\beta$ Cephei stars. If no huge amount of high precision multi-site photometric observations over an extended period is collected, ground-based photometry is clearly too limited in detection threshold to reveal all these modes. This conclusion can also be drawn from the analysis of the WIRE data of the $\delta$ Scuti star $\theta^{2}$ Tauri by Poretti et al. (2002) and the subsequent analysis of all old and new observations of this star by Breger (2002) and by Breger et al. [astro-ph/0204279].

In view of the discussion on the amplitudes of the light variations presented here, photometric mode identification of low-amplitude non-radial modes in $\beta$ Cephei stars will be very difficult without confirmation from spectroscopic variations.

Another quite fundamental conclusion is that lowamplitude modes occur in massive stars. Up to now, the preparatory programmes of future asteroseismological space missions such as MOST, MONS and COROT are focused on solar-like oscillators, roAp and $\delta$ Scuti stars. With our paper we provide evidence that there is an excellent chance of finding dense frequency spectra in $\beta$ Cephei stars. There is thus no reason to exclude massive $p$-mode pulsators from the space programmes. Including $\beta$ Cephei stars will lead to much more complete coverage of seismic targets in the HR diagram. From a theoretical point of view, $\beta$ Cephei stars have the advantage of not possessing an outer convective layer as seen in stars with masses below $2 M_{\odot}$. Moreover, their modes do not show amplitude variability - see the example of $12 \mathrm{Lac}$ in which 6 frequencies were found in photometric and spectroscopic data separated more than 10 years in time (Mathias et al. 1994). Finally, the $\beta$ Cephei stars allow us to study in detail convective overshooting and internal mixing processes due to rotation in stars that have a large inner convective core and that will eventually explode as supernovae. Observations of such stars are most likely to lead to improved evolutionary models (Maeder 2002).

Acknowledgements. We are very grateful to all those who made this use of the WIRE satellite possible. JC is grateful for support from the Belgian Fund for Scientific Research - Flanders (G.0265.97) and DB acknowledges support from NASA (NAG5-9318).

\section{References}

Aerts, C., De Pauw, M., \& Waelkens, C. 1992, A\&A, 266, 294

Aerts, C. 1996, A\&A, 314, 115

Aerts, C., De Cat, P., Cuypers, J., et al. 1998, A\&A, 329, 137 (Paper I)

Breger 2002, Comm. Asteroseismol. (Vienna), 141, 3

Buzasi, D., Catanzarite, J., Laher, R., et al. 2000, ApJ, 532, L133

Buzasi, D. 2002, in Radial and Nonradial Pulsations as Probes of Stellar Physics, ed. C. Aerts, T. R. Bedding, \& J. ChristensenDalsgaard (San Francisco ASP), ASP Conf. Ser., 259, 616

Cuypers, J. 1983, A\&A, 127, 186

Cuypers, J., Aerts, C., Buzasi, D., et al. 2002, in Proceedings of the 1st Eddington Workshop, ESA SP-485, 41

ESA 1997, The Hipparcos and Tycho Catalogues, ESA SP-1200

Heintz, W. D. 1957, Observatory, 77, 200

Heynderickx, D., Waelkens, C., \& Smeyers, P. 1994, A\&AS, 105, 447

Maeder, A. 2002, in Proceedings of the 1st Eddington Workshop, ESA SP-485, 49

Mathias, P., Aerts, C., Gillet, D., \& Waelkens, C. 1994, A\&A, 289, 875

Montgomery, M. H., \& O’Donoghue, D. 1999, Delta Scuti Newsletter, 13,28

Pagel, B. E. J. 1956, MNRAS, 116, 10

Poretti, E., Buzasi, D., Laher, R., et al. 2002, A\&A, 382, 157

Shobbrook, R. R. 1979, MNRAS, 196, 571

Van Hoof, A. 1962, ZfA, 54, 244

Wright, W. H. 1909, Lick Obs. Bull., 5, 177 